\title{
Thermo-mechanical behaviour of a Carbon Fibre Reinforced Shape Memory Alloy Hybrid Composite
}

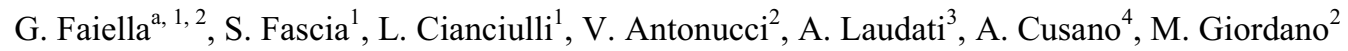 \\ ${ }^{1}$ Federico II University of Naples, Department of Materials and Production Engineering - P.le Tecchio 80, 80125 \\ Napoli, Italy \\ ${ }^{2}$ National Research Center, Institute for Composite and Biomedical Materials - IMCB - CNR - P.le Fermi 1, \\ 80055 Portici (NA), Italy \\ ${ }^{3}$ OptoSmart s.r.l. - Via Pontano, 61, 80121 Napoli, Italy \\ ${ }^{4}$ University of Sannio, Department of Engineering, Optoelectonic Division - Palazzo Bosco Lucarelli, \\ Corso Garibaldi 107, 82100 Benevento, Italy
}

\begin{abstract}
Shape memory effect, responsible of Shape Memory Alloys (SMA) shape recovering after a cycle of deforming-heating, provides to these alloys functional properties associated with the possibility of generating mechanical work simultaneously to the shape recovery.

Composite systems incorporating pre-strained SMA wires have the ability to actively change shape and other mechanical static and dynamic characteristics upon a heat driven actuation process. The functional properties of this type of adaptive composites are directly related to the reversible martensitic transformation in the SMA elements and to the constraining behaviour that the composite matrix has on the SMA wires.

In this work the thermo-mechanical behaviour of a shape memory alloy hybrid composite (SMAHC) has been experimentally investigated. A hybrid composite plate was fabricated using pre-strained SMA wires as actuating elements embedded in an thermoset resin pre-preg carbon fibres composite system. In the experimental tests, the plate was clamped at one side and actuated. During actuation, displacements will be collected using LVDT and local deformations by Bragg gratings. These optical sensors, properly attached on the plate surfaces, will be able to monitor the deformation state of the composite during the whole heating process.
\end{abstract}

\section{Introduction}

\subsection{Adaptive composites}

Adaptive composites are structural elements which can actively alter their characteristics in response to external changes. This ability is achieved by combining traditional structural materials and active materials, whose coupled behaviour provides actuating capabilities. Within this context, shape memory alloys (SMA) are ideal candidate for the design and production of adaptive structures, in which they are used as actuators. Embedding SMA elements in Fibre Reinforced Composites (FRC), used as structural matrix, it is possible create actuators which are triggered by a temperature change, thanks to the thermal shape recovery typical of SMA. Envisaged applications for shape memory alloy hybrid composites (SMAHC) include control of the structure's dynamic properties [1-2], prevention of thermal buckling [3-4] and damage repair [5].

The actuation ability of SMA elements is due to their ability of recovering pre-imposed shapes upon heating, that in constrained conditions, translates into a force exertion because the shape recovery is prevented.

Hence, pre-strained SMA elements are used as actuators embedded in composite plates, where the latter constitutes the constraint that partially prevents the shape recovery upon actuation. During heating, in fact, SMA develop recovery stresses that are transferred to the host structure, leading to a change in the shape of the hybrid composite. The magnitude of these shape changes depends on the composite stiffness and recovery stress exerted by SMA, as well as by the their positioning inside the plate. Upon an appropriate design of the hybrid composite, the control of the plate shape can be tuned by controlling the SMA temperature, because the recovery stresses depend on the temperature, in particular above the Austenite transition temperature, they increase upon an increase of the temperature. An important parameter to take into account for the SMAHC is the enhancement of

a e-mail: gabriella.faiellaeimcb.cnr.it

This is an Open Access article distributed under the terms of the Creative Commons Attribution-Noncommercial License (http://creativecommons.org/licenses/by-nc/3.0/), which permits unrestricted use, distribution, and reproduction in any noncommercial medium, provided the original work is properly cited. 
the Austenite transition temperature due to the pre-strain. This effect is known as Martensite stabilization effect and consists of an increase of the Austenite transition temperature upon an increase of the strain level imposed to the SMA element. In a previous work we reported on the effect of strain on Austenite transition temperature of a NiTi alloy, showing the existence of a linear dependence of the transition temperature on the applied pre-strain [6]. Hence, the Martensite stabilization effect shall be considered in the actuation test, in order to expect an actuation temperature higher than that related to the raw material.

In this work, the shape control of a SMAHC plate via embedded SMA wires has been experimented.

The SMAHC has been produced by using epoxy resin carbon fibres pre-impregnated composite and pre-strained NiTi wires. The experimental tests have been carried out by clamping at one side the plate and heating the SMA wires via Joule heating to trigger the shape change.

Temperature and displacement data have been recorded to characterise the thermo-mechanical behaviour of the SMAHC. Moreover, Bragg gratings have been used for the evaluation of deformation on the upper surface of the plate.

\subsection{Bragg fibre grating sensors}

Bragg fibre grating (FBG) is a particular Bragg reflector inscribed in an optical fibre in such a way to be able to reflect specific wavelengths and to transmit all others. This can be done by adding a periodic variation to the refractive index of the fibre core, so that the grating acts as a reflective (or transmission) wavelength filter. The period of the induced refractive index modulation in the optical fibre core determines the wavelengths that will be reflected. When the fibre grating is subjected to external stimuli, such as strain and temperature, its period changes, causing in turn a reflective wavelength change. Fibre grating period change is induced by both changes in the length of grating period and in the core refractive index. The result of the combination of these two effects is an overall change of the period grating, consequently resulting in a shift in the reflected wavelength. This wavelength shift is used for the measurement of strain and temperature. In Fresnel reflection the light travelling between media having different refractive indices can both reflect and refract the light at interface. The reflected wavelength depends on the fibre refractive index and is a function of the grating period too, as follows :

$$
\lambda_{\mathrm{B}}=2 \mathrm{n}_{\mathrm{eff}} \Lambda
$$

where $\lambda_{B}$ is the reflected wavelength, $n_{\text {eff }}$ is the effective grating refractive index, $\Lambda$ is the period of the fibre grating [7].

\section{SMAHC production and characterization}

The aim of the SMAHC actuation is to control the bending behaviour of a thin plate, thus the SMA elements are pre-strained and positioned at a distance with respect to the plate midplane. The expected shapes in the cold and hot configurations are reported in figure 1.

COLD

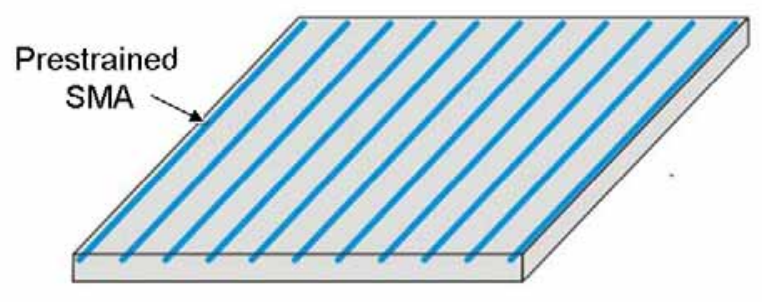

HEATED

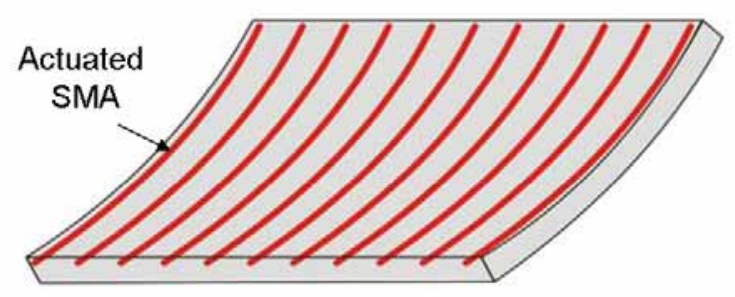

Fig. 1. SMAHC cold and hot configurations

The integration of SMA elements in FRC is particular easy, hence they constitute a particularly suitable type of host structure. The manufacturing technique of the composite is very important for the SMAHC design, because the maximum temperature reached during the thermoset resin curing process is a fundamental aspect to be taken into account for the choice of SMA materials. The imposed pre-strain must be maintained in the SMA elements upon curing, so if the resin curing temperature is higher than the Austenite transition temperature, SMA elements have to be blocked in strained condition during the composite production, in order to prevent the pre-strain loss. Another possibility consists of using a resin whose curing temperature is lower than the Austenite transition temperature, so that the SMA elements can be positioned in the composite without the need on any blocking system. Among all the composites processing techniques, the use of resin pre-impregnated (pre-preg) fibres is particular advantageous for SMAHC production, because it enables to handle with pre-constituted materials, 
avoiding the use of dry fibres and liquid matrix, that could cause problems related to resin flowing and fibres wettability. A fundamental parameter to take into account in SMAHCs operating is the good load transfer between the SMA elements and the host structure. In order to enhance it, etching treatments or mechanical abrasion could be performed on the SMA elements before the embedding.

\subsection{Materials and techniques}

In this work, an epoxy resin carbon fibres pre-preg having a curing temperature higher than the Austenite transition temperature is used, so the use of a blocking system is necessary for SMA wires.

A steel blocking plate equipped with an integrated straining system has been used to deform SMA wires at a desired strain level and to block them during the curing.

Trained Nitinol wires of $0.5 \mathrm{~mm}$ in diameter were provided by Saes-Getters. Transition temperatures measured using Differential Scanning Calorimetry (DSC TA Instruments 2920) are reported in figure 2 and summarized in table 1. A small amount of R-Phase can be detected during the cooling scan between 70 and $50^{\circ} \mathrm{C}$. The actual Austenite transition temperatures are those related to the second heating cycle, because the first one is necessary to cancel the mechanical effects due to the fabrication (table 1).

The Austenite transition temperature of the strained wire has been measured, as well, in order to take into account the Martensite stabilization effect induced by the straining process. The transition temperature of the wire strained at $3.5 \%$ is $104^{\circ} \mathrm{C}$.

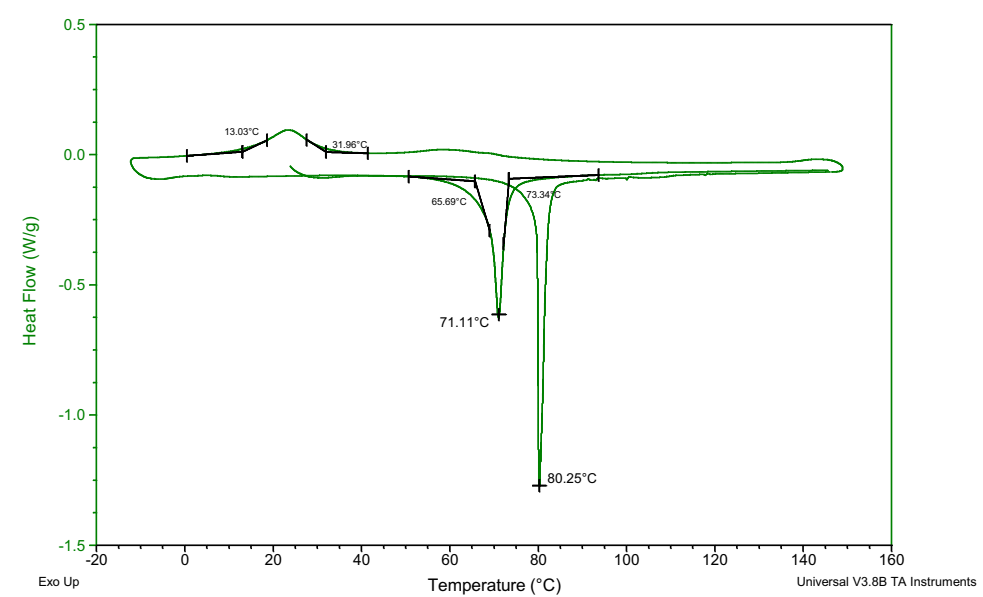

Fig. 2. Differential Scanning Calorimetry heat flow vs. temperature curve of the NiTi wire

Table 1. NiTi transition temperatures evaluated from DSC curve

\begin{tabular}{|c|c|c|c|}
\hline $\mathrm{A}_{\mathrm{s}}\left[{ }^{\circ} \mathrm{C}\right]$ & $\mathrm{A}_{\mathrm{f}}\left[{ }^{\circ} \mathrm{C}\right]$ & $\mathrm{M}_{\mathrm{s}}\left[{ }^{\circ} \mathrm{C}\right]$ & $\mathrm{M}_{\mathrm{f}}\left[{ }^{\circ} \mathrm{C}\right]$ \\
\hline 66 & 71 & 32 & 13 \\
\hline
\end{tabular}

An unidirectional carbon fibres epoxy pre-preg has been used as host structure for embedding SMA wires.

The lamination sequence used for the fabrication of the SMAHC is $(90 / 90 / 0 / 90 / 90)$, where the $0^{\circ}$ direction is referred to the one parallel to the SMA wires. The plies have been layered in order to have low stiffness in the direction of the SMA wires. These have been placed between the first and the second layer of pre-preg, in order to have the maximum possible distance between the midplane and the wires. The wires have been placed at 15 mm spaced in-plane. The final dimensions of the SMAHC were $150 \times 75 \mathrm{~mm}$, with a thickness of $1.7 \mathrm{~mm}$. The entire lay up has been performed directly on the steel plate, using a release agent to allow the detachment of the cured final composite. All wires have been mechanical abraded to enhance the adhesion with the pre-preg.

The wires have been pre-strained at a strain level of 3.5\% using the straining system equipped on the frame and then allowed to recover the elastic deformation before the final blocking. The deformation value has been chosen inside the pseudoelastic plateau range. Recovery stress tests have been performed on the wire deformed at a $3.5 \%$ strain. The measured recovery stresses exerted by the wire at 80 and $160^{\circ} \mathrm{C}$ are respectively 120 and $430 \mathrm{MPa}$. After the complete lay up, a release film and a vacuum bag have been placed on the top of the layers. The vacuum has been pulled in order to facilitate a first layers attachment. Still pulling the vacuum, the plate has been placed on the plate of the hot press, where the proper curing cycle had been programmed. An initial pressure of 2 bar has been applied for 10 seconds at room temperature, than a constant pressure of 4 bar has been applied during all the curing cycle. The latter consists of a heating ramp at $2^{\circ} \mathrm{C} / \mathrm{min}$ from room temperature to $120^{\circ} \mathrm{C}$, a constant temperature step for 1 hour and a cooling ramp to room temperature at $2^{\circ} \mathrm{C} / \mathrm{min}$. 


\subsection{SMAHC actuation}

\subsubsection{Experimental set-up}

A first attempt of actuation by means of an external heating source has been done, using a heating plate under the composite. However, the actuation cannot be tuned because of the big difference in temperatures established during the heating between the surface closer to the plate and the upper one.

Hence, an electrical heating system has been designed in order to allow a current to circulate in the SMA wires sufficient to heat them. SMA wires have been connected in parallel and a power supplier (HQ Power adjustable DC power supply PS 3020) has been used in order to force enough current in the wires, taking into account its dispersion in the conductive carbon fibres. An outline of the experimental setup is shown in figure 3 . The composite has been clamped on one side, leaving a free length of $125 \mathrm{~mm}$ in such a way that the side of the panel closer to the wires is the under face, hence during SMA actuation the panel will down inflect. The displacement in a point on the free side (point A in figure 3) has been monitored using an LVDT (DFg5 unguided min LVDT, $\pm 5 \mathrm{~mm}$ stroke).

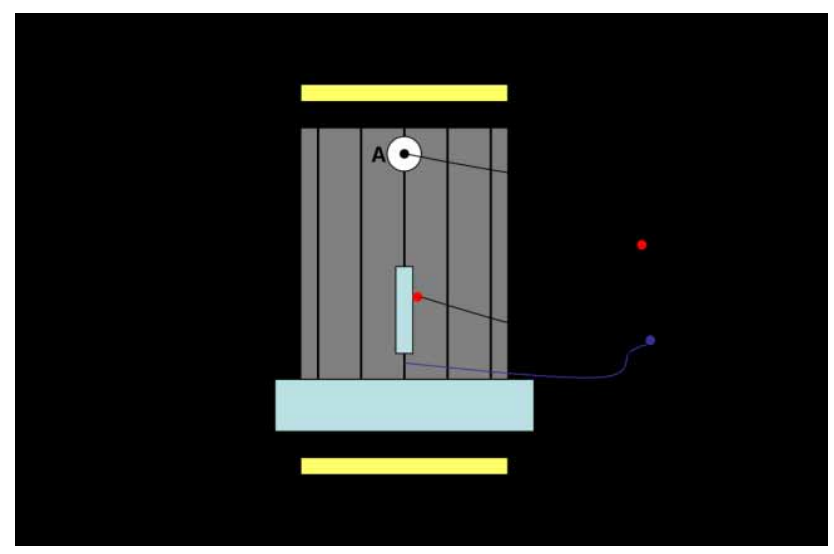

Fig. 3. Experimental set-up

A Bragg grating sensor has been attached on the upper surface of the SMAHC plate using a commercial cyanoacrylate adhesive. A K type thermocouple monitoring the temperature of the panel has been placed just near the Bragg sensor, in order to take into account the temperature contribution on the sensor signal during the actuation test. This temperature will be considered as a reference in the actuation test graph. The maximum operating temperature that can be reached on that plate is $95^{\circ} \mathrm{C}$, because a higher temperature could induce a detachment of the sensor from the plate. Moreover, the glass transition temperature of the epoxy resin matrix has been measured to be $97^{\circ} \mathrm{C}$.

Another thermocouple has been attached on the wires outside the composite. However, an important aspect to be noted is that the temperature of the wires inside the plate, that are the ones that actuate the SMAHC, is lower than that measured on the wires outside it, because part of the current applied to the wires flows in the carbon fibres. The system SMA wires/carbon fibres shall be view as a parallel of two resistances whose entity cannot be evaluated, because the variation of one of them will cause the variation of the other one. Hence, the real temperature value of the wires responsible for the actuation cannot be evaluated, but is certainly higher than the one measured on the upper surface, that has been monitored.

\subsubsection{Experimental results}

Figure 4 shows a profile picture of the SMAHC specimen (a) at room temperature and (b) at $90^{\circ} \mathrm{C}$, where a significant deformation is observed. As it can be seen, at room temperature the plate is flat, while at $90^{\circ} \mathrm{C} \mathrm{SMA}$ actuation causes the deflection of the plate.

Figure 5 shows graph reporting the displacement as a function of the panel temperature related to the first actuation of the SMAHC. In this plot it is possible to note that the maximum displacement reached at $90^{\circ} \mathrm{C}$ is about $9 \mathrm{~mm}$. At the end of the cooling step a non recovered displacement of abut $3.5 \mathrm{~mm}$ can be observed. 

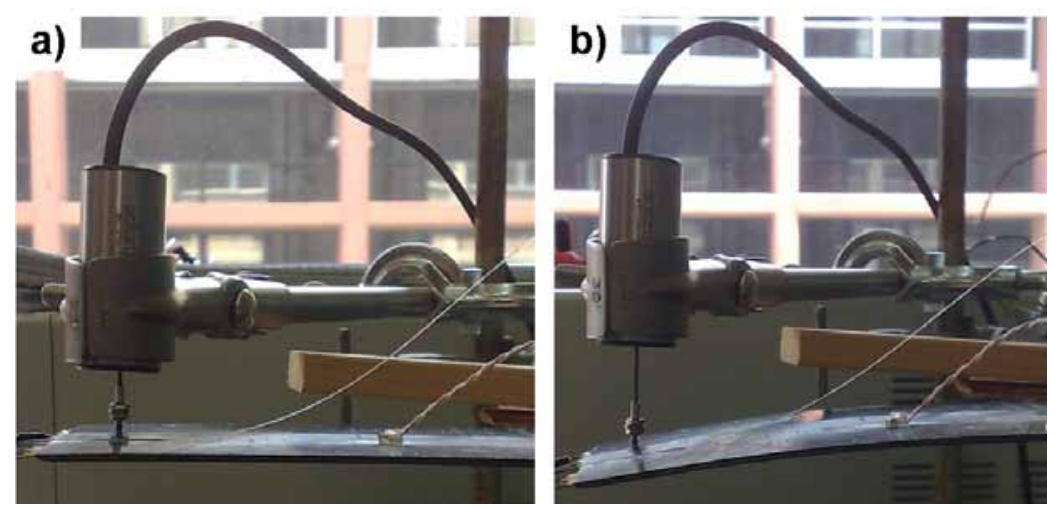

Figure 4. SMAHC plate a) at room temperature and b) at $90^{\circ} \mathrm{C}$

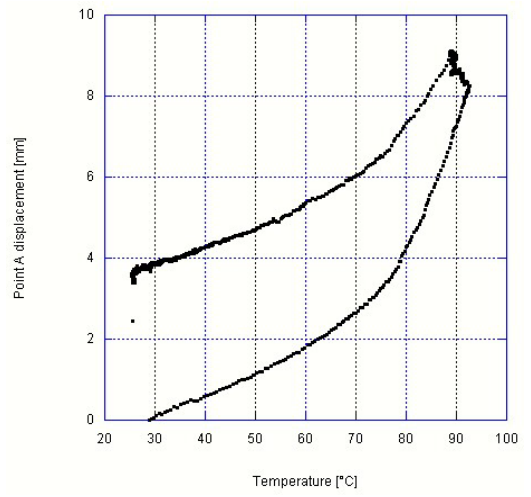

Fig. 5. Displacement vs. plate temperature during first actuation

In figure 6, the deformation calculated from the Bragg data acquired during the first actuation is plotted against the temperature. The deformation, $\varepsilon$, has been calculated by subtracting the temperature contribution from the registered shift of the Bragg wavelength, in order to avoid in the final calculated deformation values thermal deformation of the grating and those due to the composite expansion.

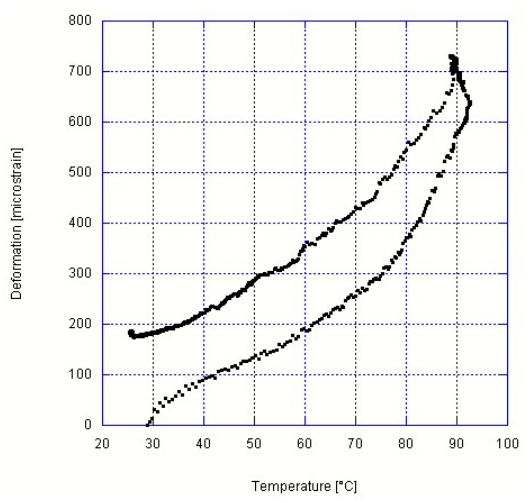

Fig. 6. Deformation vs. temperature

The deformation reported in figure 6 shows the same trend of the displacement with the temperature, with a maximum deformation of $700 \mu$ strain at $90^{\circ} \mathrm{C}$. As expected, the upper surface of the composite is subjected to a tensile state since the deformation is positive during all the actuation test.

In order to test the reproducibility of the experiment, several actuations have been performed on the SMAHC. In figure 7 the deformation related to the second heating-cooling test is reported against the temperature. 


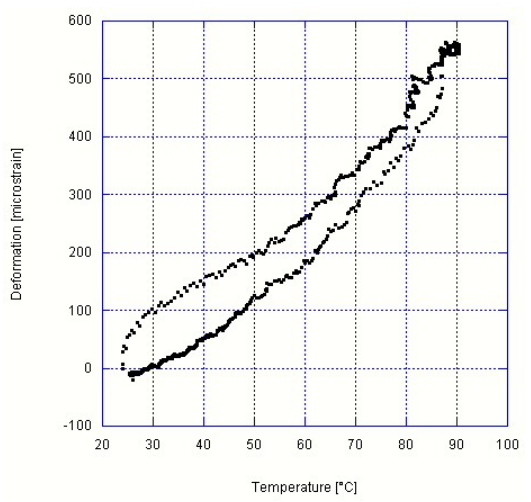

Fig. 7. Deformation evolution with temperature during the second actuation test

In the second actuation test, the maximum deformation reached at $90^{\circ} \mathrm{C}$ is lower than in the first actuation cycle. This is due to a loss in actuation ability of the SMA, confirmed by the displacement vs. temperature graphs reported in figure 8 , where the maximum displacements reached at $90^{\circ} \mathrm{C}$ are lower than the one reached in the first actuation test and all cycles are reproducible.

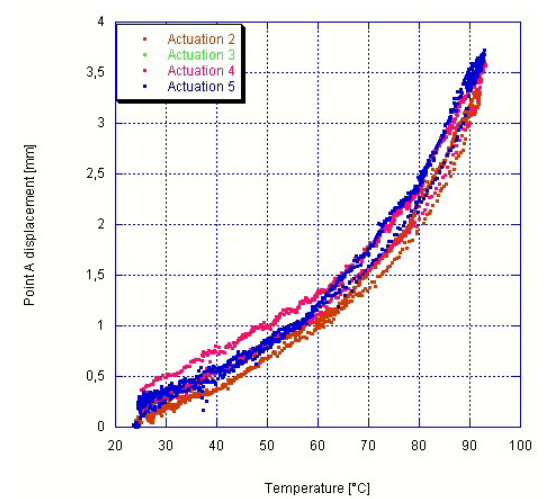

Fig. 8. Displacement vs. plate temperature during various actuation tests

In order to assess the decreased actuation ability of the embedded SMA wires, a calorimetric analysis has been carried out on a SMA sample extracted from the tested SMAHC, so that it is possible to compare its Austenite transition temperature with the one of the pre-strained wire before blocking and actuation (fig. 9).

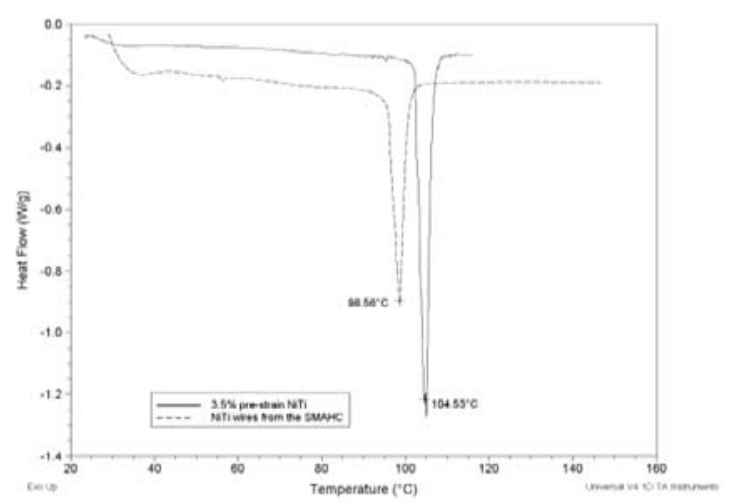

Fig. 9. Heat flow vs. temperature curves of $3.5 \%$ pre-strained wire and SMA sample extracted from the tested SMAHC

A decrease in the transition temperature of the SMA sample extracted from the SMAHC can be detected from 104 to $98^{\circ} \mathrm{C}$. This decrease is due to a partial loss of the pre-strain in the actuated wire. The transition temperature is, in fact, intermediate between undeformed and the $3.5 \%$ strained wire.

\section{Conclusions}

The fabrication and the actuation of a SMAHC plate constituted by epoxy carbon fibres pre-preg and SMA wires have been carried out. Displacements at the free end and local deformations at the mid of the upper surface have been measured during the actuation test. They show the same evolution with temperature, assuming the 
maximum values during the first actuation cycle and lower values for the subsequent cycles. This effect has been shown to be related to the loss of pre-strain in the SMA elements during the actuation, probably due to a partial debonding between wires and matrix. Future works will focus on the fabrication and testing of SMAHC embedding Bragg grating sensors on different plies, in order to measure the deformation state in plane and also along the thickness of the plate.

\section{References}

[1] J. Ro, A. Baz, Compos. Eng. 5, p.91 (1995)

[2] A. Zak, M. Cartmell, W. Ostachowicz, Key Eng Mat., 233 (2003), pp. 245-246

[3] J. Ro, A. Baz, Compos. Eng. 5, p.77 (1995)

[4] V. Birman, Int. J. Mech. Sci. 39, p.1139 (1997)

[5] D. S. Burton, X. Gao, L. C. Brinson, Mech. Mater. 38, p.525 (2006)

[6] G. Faiella, V. Antonucci, M. Giordano, F. Daghia, E. Viola, Advances in Science and Technology 59, 57 (2008)

[7] K. O. Hill, Y. Fujii, D.C. Johnson, B. S. Kawasaki, Appl. Phys. Lett. 32, p.647 (1978) 